\title{
Flex-based Integrated Monitoring System in Computer Rooms
}

\author{
Kehang Xu' ${ }^{1}$, Bo Su${ }^{1}$, XiaoWei Han ${ }^{1}$, Qin Jing ${ }^{2}$ \\ 1. SouthWest China Branch of State Grid Corporation of China, Sichuan 620010 \\ 2. Sichuan Grid Power Communication Technology Co,.Ltd, \\ Sichuan 620010
}

Key words: information devices; data of power equipment and environment; centralized monitoring

Abstract:The paper firstly introduces the core tech related to the integrated monitoring system in computer rooms and then summarizes the problems hidden in the system. At last, the paper aims at the elaboration of the design of a new Flex-based integrated monitoring system in computer rooms from tech selection, system structure and system functions and so on.

\section{System requirement}

Most of monitoring systems in computer rooms are J2EE-based which involve multiple levels and much complexity. Although the application servers and database are capable of real-time monitoring in a certain degree, they are inadequate in monitoring the Web application operation; Besides, the monitored information in each level fails to be connected with each other in an efficient manner, in other words, the system administrator is not able to have an access to the overall operation of the program in web operation. In practice, the commonly used computer room monitoring system operation is shown as below. ${ }^{[1]}$

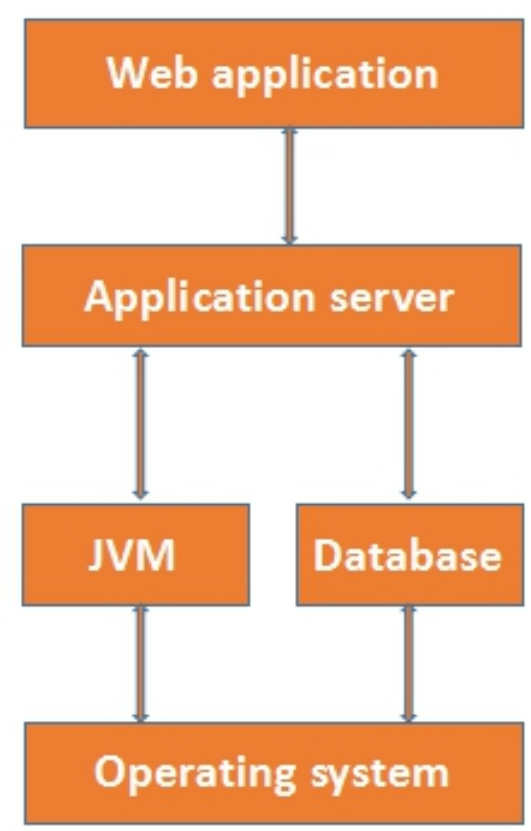

Fig.1 Schematic diagram of the operating environment of the traditional computer room monitoring systems

In general, the cluster environment for the online $\mathrm{J} 2 \mathrm{EE}^{[2]}$ in production is constructed by two or more application servers and database servers, so a comprehensive response to the application operation is in need of processing and analyzing the monitoring information at all nodes in the cluster environment in a unified manner.

From the above analysis, it can be seen that the effective monitoring of J2EE application is 
extremely complex and difficult and thus a new computer room integrated monitoring system is yet to be designed.

\section{Design for Flex-based computer room integrated monitoring system}

\section{Related technology}

(1) Flex. Flex is a programmatic framework for RIA development which enables the effective integration of the optimal user interface of desktop applications, a wide use of Web application and the cost-effect and interactive multimedia, and is thus rated as a powerful tool for the research and development of a new generation of Web application.

Flex framework ${ }^{[3]}$ mainly involves $\mathrm{MXML}^{[4]}$ page markup language, Flex class library and ActionScript programming language among which MXML page markup language is based on XML with the main functions of arranging the interface and the control display; the user interface and the server data are bound via MXML so that the complexity of data access programming is greatly reduced and the efficiency of programming is greatly enhanced; Flex class library as a core is in a position to provide a series of controls and components to make sure of a better display of the user interface; ActionScript as an object language is mainly aimed at having a control over the GUI components, data callback and monitoring.

(2) JMS technology ${ }^{[5]}$. JMS technology mainly targets at the definition of the standard interface and grammar of the client and JMS providers. It belongs to J2EE made up of management object, connection, session, message, message compilers and consumers and focuses on synchronized or asynchronous communication between clients and computers.

JMS technology takes its best advantage of open standards and the correctly-designed codes, capable of ensuring that all JMS servers are compatible with the system; in addition, JMS technology can do a good job in the message transmission, and it is able to send the actually sent message to the JMS server and then to the addressed destination. As long as the message code to the server is correct, the message can be still accessed even if the server goes wrong in the process.

\section{System Structure}

From the above description and analysis, it can be seen that J2EE-based Web application needs to deal with two crucial problems namely, performance and applicability. In the former, the key problem that the previous system in a normal operation is impacted due to the increase of the monitoring systems should be tackled; in terms of applicability, the monitoring system should be deployed and operated efficiently in the application system so that the values of the monitoring system can be given a full play. The basic structure is shown in Fig.2:

The normal communication between the monitoring server and monitoring agent in the Flex-based computer room integrated monitoring system is mainly achieved by JMS. The main functions of JMS are manifested as follows: it can further improve the data processing ability of the computer room integrated monitoring system; JMS itself has its own queue and theme subscription function, especially catering for the cluster environment most in that it can achieve monitoring by many computers at the same time; usually the monitoring system will resort to visualized means such as charts and so on to display the relevant data; In addition, the Flex technology can be applied to the monitoring information display level so that a more efficient and a simple user interface can be provided for the sake of smooth interaction between the system and users. 


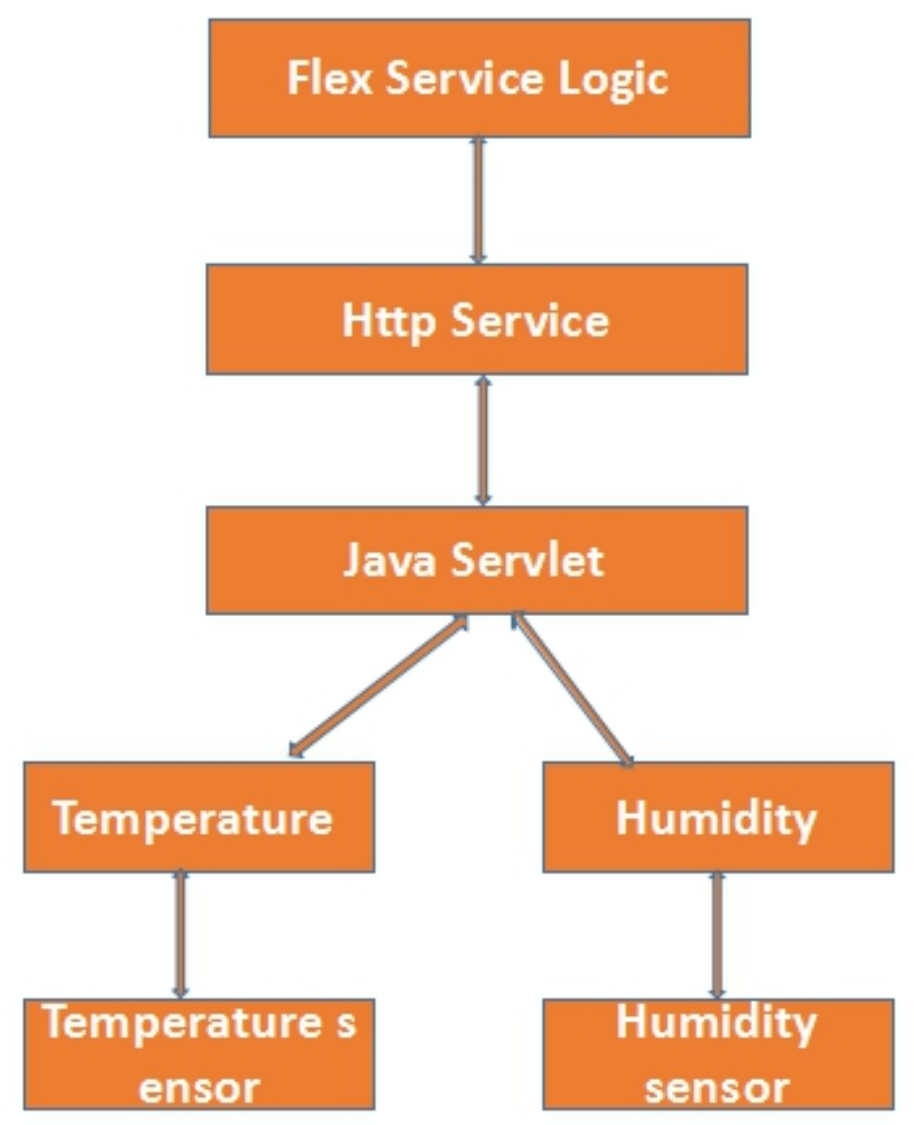

Fig. 2 Drawing of system structure

Combined with the above description and analysis, the present writer has the design proposal on the flex-based computer integrated monitoring system which mainly involves four levels, monitoring resources, monitoring agents, monitoring messages and monitoring services in a bottom-up order. The monitoring agent mainly runs in each node of the cluster, the monitoring agent and the monitoring service are independent without influence from each other. This design can ensure that the monitoring system can run well in the cluster environment, thus effectively solving the applicability problem mentioned in the above on the introduction of the tech choice. The monitoring agent has the function of data collection instead of the specific monitoring while the specific monitoring work mainly falls on the monitoring server and the reason of this aims to effectively solve the above mentioned performance problems. The monitoring server summarizes and processes the data collected by the system monitoring agent so as to have an effective control over the impact of system monitoring on the application. Meanwhile, JMS-based technology monitoring message is used to complete the decoupling between the monitoring agent and the monitoring service center. Besides, the custom message format is selected so that both of the network communication efficiency and the overall performance of the monitoring system can be improved further.

\section{System function}

The related module functions of the application monitoring system are shown in the following table. 
Table 1 The related module functions of the application monitoring system

\begin{tabular}{|l|l|}
\hline components & Description \\
\hline $\begin{array}{l}\text { Monitoring } \\
\text { resources }\end{array}$ & $\begin{array}{l}\text { Monitored host or program is mainly responsible for providing data indicators } \\
\text { collected by the monitoring system }\end{array}$ \\
\hline Monitoring agent & $\begin{array}{l}\text { It is distributed among the monitored objects, takes charge of collecting various } \\
\text { data indicators, achieves the message communication by monitoring messages } \\
\text { and monitoring services and completes the specific monitoring operation }\end{array}$ \\
\hline $\begin{array}{l}\text { Monitoring } \\
\text { message }\end{array}$ & $\begin{array}{l}\text { The monitoring message is between the monitoring agent and the monitoring } \\
\text { server, mainly responsible for to be responsible for decoupling of } \\
\text { communication between the two. It provides a unified messaging interface for } \\
\text { the monitoring server so that a smooth two-way communication can be done } \\
\text { between the two. }\end{array}$ \\
\hline $\begin{array}{l}\text { Monitoring } \\
\text { services }\end{array}$ & $\begin{array}{l}\text { Monitoring service as a program in the monitoring system management aims at } \\
\text { the parameter configuration of collected data at each monitoring point, statistics } \\
\text { and analysis of the collected, measurement and evaluation of the system } \\
\text { operating indicators to generate a system performance analysis report so that an } \\
\text { alarm can be issued timely in the event of data going beyond the threshold } \\
\text { values. }\end{array}$ \\
\hline
\end{tabular}

The core of the flex-based computer room integrated monitoring system lies in the monitoring service. The main functions of the monitoring service are as follows:

The first rests in the data processing. Monitoring server and database server are responsible for processing the specific data, that is, classifying and processing the received real-time data from the data collection modules in accordance with the specific requirements. Meanwhile, they also save processed data in the database server facilitating the monitoring system to query the relevant real-time data.

The classification, processing, storage of all data from the collection modules and the interaction between all data in the system is carried out in the database server. The monitoring server is not equipped with the data storage mechanism and corresponding devices, so the relevant data can be only saved in the database server.

The second lies in monitoring and management. Specifically, it resorts to the monitoring console for the configuration and management of the monitoring agent and adjusts the monitoring agent during the operation to ensure that the monitoring adjustment can be achieved in the process of the system operation. The monitoring management process is shown in the following figure.

The third is the threshold alarm. The so-called threshold alarm means that the monitored abnormal real-time data and historical data in case of abnormal threshold can be sent to users accurately and timely so that users can engage in timely adjustment in the system or related business with a consideration of the actual situation thereby effectively avoiding the system operating efficiency decline and the system disorders or even system crashes and so on. Threshold alarm is realized as below: 


\section{Start}

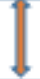

Administrators use the functions

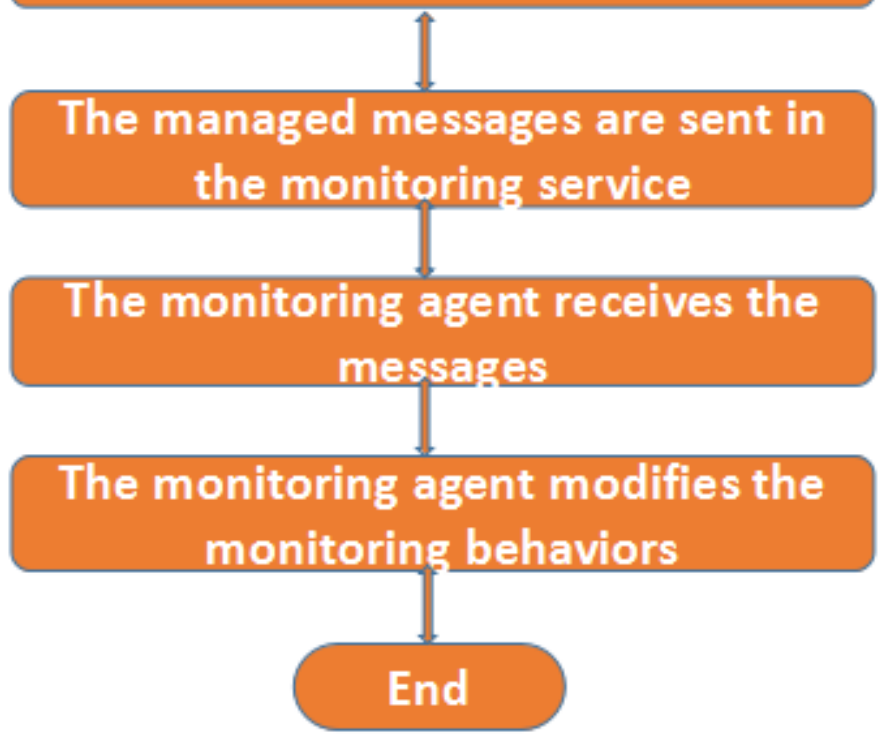

Fig.3 The basic flow chart of the monitoring

\$temp = array (

'monitor_header' => array （ . ) ， 'monitor_sound' => array （）, 'memory_sound' => $\quad$ array ( 'friendly_name' => ' Memory out Alarm Voice ' , 'method' => 'drop_array' , 'the alarm sound goes beyond the memory' 'array' =>monitor_scan_dir ( ) , 'default' => 'memory-sound.mp3' , )

The notices in the threshold warning can be divided into the following: Firstly, the alarm information is sent to the alarm processing module to remind the user to deal with it in a timely manner; Secondly, the alarm information is displayed with data or images to remind users of the abnormal data in the system; Finally, the Web interface is used to browse the alarm information for an access of the abnormal data which is based on to judge whether the abnormal data attribute to the network connection error or an application exception.

Fourthly, the data display is mainly based on the unified view provided by the application so as to provide users with the specific data on the business they care for to facilitate them to solve the performance and availability issues. For the majority of users, the data display can be helpful to provide a staggering array of monitoring data so that users can have a knowledge of the system history and the current operating conditions easily.

\section{Conclusion}

To sum up, the present writer puts forward a design of Flex-based room integrated monitoring system in view of the insufficiency in the current commonly used computer room integrated monitoring system. The practice indicates that the system features real-time application, stability, reliability, fault tolerance and universal applicability and is able to solve the concurrent control of many users without excessive dependence on a certain established application services or message servers. In general, the system can be effectively operated on other servers without special adjustments. In addition, it can run on other servers in an efficient manner without specific adjustment. Moreover, the system is integrated with a loose coupling 
to ensure of its superior reuse performance. Thus, the flex-based the computer room integrated monitoring system in this paper is of great value in practical application and promotion.

\section{Reference}

[1] Praveen Gupta,Prof. M.C.Govil.Spring Web MVC Framework for rapid open source J2EE application development: a case study[J].International Journal of Engineering Science and Technology,2010,2(6):1684...

[2]IEC60950-23-2005IEC60950-23 , Ed.1.0 : Part 23, Information technical device. Security: Large data storage equipment [S].

[3] MANN H B, Non-parametric Test Against Trend [J]. Econometrical, 1945,13:245-259.

[4]Operation and maintenance working rules of State Grid information system

[5]E.W.L. Cheng,H. Li. Utility of consistency measure in the analytic hierarchy process[J]. Construction Innovation: Information, Process, Management, 2003,3(4). 